\title{
MicroBlack Holes Thermodynamics in the Presence of Quantum Gravity Effects
}

\author{
H. Soltani, ${ }^{1}$ A. Damavandi Kamali, ${ }^{2}$ and K. Nozari ${ }^{3}$ \\ ${ }^{1}$ Department of Physics, Islamic Azad University, Nour Branch, Nour, Iran \\ ${ }^{2}$ Department of Physics, Islamic Azad University, Science and Research Branch, Tehran, Iran \\ ${ }^{3}$ Department of Physics, Islamic Azad University, Sari Branch, Sari, Iran
}

Correspondence should be addressed to K. Nozari; knozari@umz.ac.ir

Received 5 November 2013; Accepted 18 February 2014; Published 1 April 2014

Academic Editor: George Siopsis

Copyright (C) 2014 H. Soltani et al. This is an open access article distributed under the Creative Commons Attribution License, which permits unrestricted use, distribution, and reproduction in any medium, provided the original work is properly cited. The publication of this article was funded by SCOAP ${ }^{3}$.

Black hole thermodynamics is corrected in the presence of quantum gravity effects. Some phenomenological aspects of quantum gravity proposal can be addressed through generalized uncertainty principle (GUP) which provides a perturbation framework to perform required modifications of the black hole quantities. In this paper, we consider the effects of both a minimal measurable length and a maximal momentum on the thermodynamics of TeV-scale black holes. We then extend our study to the case that there are all natural cutoffs as minimal length, minimal momentum, and maximal momentum simultaneously. We also generalize our study to the model universes with large extra dimensions (LED). In this framework existence of black holes remnants as a possible candidate for dark matter is discussed. We study probability of black hole production in the Large Hadronic Collider (LHC) and we show this rate decreasing for sufficiently large values of the GUP parameter.

\section{Introduction}

All approaches of quantum gravity support the idea that near the Planck scale, the standard Heisenberg uncertainty principle should be reformulated by the so-called generalized uncertainty principle (GUP) [1-3]. In particular, string theory [4], loop quantum gravity [5], noncommutative geometry [6], and the TeV black hole physics all indicate the existence of a minimum observable length [7]. Also black hole Gedanken experiments support this idea in a fascinating manner $[8,9]$. Incorporation of gravity in quantum field theory naturally leads to an effective cutoff (a minimal measurable length) in the ultraviolet regime. In fact, the high energies used to probe small distances significantly disturbing the spacetime structure by their powerful gravitational effects.

Within the standard view of black hole thermodynamics, based on the entropy expression of Bekenstein and the temperature expression of Hawking, a small black hole should emit black body radiation, thereby becoming lighter and hotter gradually leading to an explosive end when the mass approaches zero. But it has been argued that the generalized uncertainty principle prevents a black hole from complete evaporation [10-24]. On the other hand, a version of the GUP with higher orders in the Planck length induces quantitative corrections to the entropy and then influences the Hawking evaporation of black holes. Then, the ultimate quantum nature of the physics at the Planck scale would be best described in the framework of a GUP containing the gravitational effects to all orders in the Planck length.

The idea of large extra dimensions (LEDs) which recently has been proposed, might allow studying interactions at Trans-Planckian energies in the next generation collider experiments. The model of Arkani-Hamed, Dimopoulos, and Dvali (ADD) [25] used $d$ new large space-like dimensions without curvature, and gravity is the only force which propagates in the full volume of the space-time (the bulk). Hence, the gravitational force in the four-dimensional world (the brane) appears weak compared to the other forces which do not propagate in the extra dimensions.

Among the predicted effects, the experimental production of black holes at particle colliders such as the Large Hadronic Collider (LHC) is one of the most exciting 
possibilities which has received a great amount of interest. Therefore, black holes may even be observed in current or near-future experiments. Possible production and detection of TeV-scale black holes in colliders such as the LHC provide a suitable basis to test our comprehending of black hole physics at Planck scale and quantum theory of gravity itself. For extra-dimensional gravity at $\mathrm{TeV}$-scale, this leading order term in the expansion of corrected entropy relation leads to a significant change in the possibility of formation and detection of TeV black hole in the laboratories such as the LHC (see, for instance, [26-38]).

Until now all of the work has been done with GUP in the leading order in the fundamental length. On the other hand, it has been considered, in the context of doubly special relativity (DSR) $[39,40]$, that a test particle's momentum cannot be arbitrarily imprecise and therefore there is an upper bound for momentum fluctuations. So, there is also a maximal particles momentum. Existence of an upper bound for the test particles momentum provides several novel and interesting features, some of which are studied in [41-45].

In this paper, we are going to study the effects of natural cutoffs encoded in GUPs on the thermodynamics of microblack holes in two stages: firstly we consider a GUP that admits just a minimal length and maximal momentum and we call it GUP* and secondly a GUP that admits a minimal length, a minimal momentum, and maximal momentum and we call it GUP**. We study thermodynamics of $\mathrm{TeV}$-scale black holes in a model universe with large extra dimensions (LED) in the context of these GUPs. In this framework, the corrections to black hole thermodynamic parameters may have important consequences on the black hole production at particle colliders. We compare the results obtained by each of the above mentioned GUPs and we focus on the role of natural cutoffs in this framework. Existence of black holes remnants as a possible candidate for dark matter is discussed also.

The organization of this work is as follows: in Section 2, we introduce a generalized uncertainty principle with minimal length and maximal momentum (GUP*) and also a generalized uncertainty principle which admits a minimal length, a minimal momentum, and maximal momentum $\left(\mathrm{GUP}^{* *}\right)$. In Section 3, we obtain an expression for the black holes temperature with GUP* and we calculate entropy and heat capacity of black hole with this GUP. In Section 4, the GUP $^{* *}$-corrected thermodynamic parameters are computed. The paper follows by summary and discussion in Section 5 .

\section{A Brief about GUP}

2.1. Generalized Uncertainty Principle with Minimal Length and Maximal Momentum $\left(G U P^{*}\right)$. It has been considered in the context of the doubly special relativity (DSR) that a test particle's momentum cannot be arbitrarily imprecise and therefore there is an upper bound for momentum fluctuations [46-54]. Then, it has been shown that this may lead to a maximal measurable momentum for a test particle $[39,40]$. In this framework, the GUP that predicts both a minimal observable length and a maximal momentum can be written as follows $[39,40]$ :

$$
\Delta x \Delta p \geq \frac{\hbar}{2}\left(1-2 \alpha\langle p\rangle+4 \alpha^{2}\left\langle p^{2}\right\rangle\right)
$$

or

$$
\Delta x \Delta p \geq \frac{\hbar}{2}\left[1-\alpha(\Delta p)+2 \alpha^{2}(\Delta p)^{2}\right]
$$

This relation can lead us to the following commutator relation (see [39]):

$$
[x, p]=i \hbar\left(1-\alpha p+2 \alpha^{2} p^{2}\right)
$$

where $\alpha$ is a positive constant in the presence of both minimal length and maximal momentum. In LEDs scenario and based on the ADD model, GUP can be written as follows:

$$
\Delta x_{i} \Delta p_{i} \geq \frac{\hbar}{2}\left(1-\alpha L_{P}\left(\Delta p_{i}\right)+2 \alpha^{2} L_{P}^{2}\left(\Delta p_{i}\right)^{2}\right),
$$

where the Planck length in a model universe with large extra dimensions is defined as $L_{P}=\left(\hbar G_{d} / c^{3}\right)^{1 /(d-2)}$ and $G_{d}$ is gravitational constant in $d$-dimensional space-time. In the ADD scenario, $G_{d}=G_{4} L^{d-4}$, where $L$ is the size of the extra dimensions. By saturating the inequality in (4) and solving for $\Delta p_{i}$, we find

$$
\Delta p_{i}=\left(\frac{\alpha L_{P}+2 \Delta x_{i}}{4 \alpha^{2} L_{P}^{2}}\right)\left(1-\sqrt{1-\frac{8 \alpha^{2} L_{P}^{2}}{\left(\alpha L_{P}+2 \Delta x_{i}\right)^{2}}}\right),
$$

so that the minimal position uncertainty has the value

$$
\Delta x_{i} \geq \Delta x_{\min }=\alpha L_{P}\left(\frac{2 \sqrt{2}-1}{2}\right) .
$$

This result can be described as a new minimal length scale.

2.2. Generalized Uncertainty Principle with Minimal Length, Minimal Momentum, and Maximal Momentum $\left(G U P^{* *}\right)$. In this section, we consider a generalized uncertainty principle that admits a minimal length, a minimal momentum, and maximal momentum. The minimal length as usual comes from the finite resolution of space-time points (space-time fuzziness) in Planck scale. In fact it is based on the fact that a string cannot probe distances smaller than its length. Maximal momentum, as we have explained previously has its origin in the doubly special relativity. Actually, existence of a minimal fundamental length naturally leads to the existence of maximal energy for test particle and hence the maximal momentum. Existence of a minimal momentum in some sense needs more explanation: it is known that for large distances, where the curvature of space-time becomes important, there is no notion of a plane wave on a general curved space-time [55] (see also [56]). This means that there appears a limit to the precision with which the corresponding momentum can be described. One can express this as a nonzero minimal uncertainty in momentum measurement. 
For example, by an intuitive procedure relying on harmonic oscillator, it is known that quantum mechanical ground state energy of a harmonic oscillator is not zero and has a minimum value. So one can conclude the smallest uncertainty in momentum is not zero. This smallest uncertainty in momentum measurement can be considered nontrivially as the minimal momentum. In fact, this is a nontrivial assumption that minimal ground state energy in harmonic oscillator should quantum theoretically be described as a nonzero minimal uncertainty in momentum measurements (for more details see $[57,58]$ ). Based on these arguments, one infers the following expression:

$$
\Delta x \Delta p \geq \frac{\hbar}{2}\left[1-2 \alpha(\Delta p)+4 \alpha^{2}(\Delta p)^{2}+4 \beta^{2}(\Delta x)^{2}\right]
$$

We can write the following relation in extra dimensions as

$$
\begin{aligned}
& \Delta x_{i} \Delta p_{i} \\
& \quad \geq \frac{\hbar}{2}\left(1-2 \alpha L_{P}\left(\Delta p_{i}\right)+4 \alpha^{2} L_{P}^{2}\left(\Delta p_{i}\right)^{2}+4 \beta^{2} L_{P}^{2}\left(\Delta x_{i}\right)^{2}\right) .
\end{aligned}
$$

This relation leads to a nonzero minimal uncertainty in both position $\Delta x_{0}$ and momentum $\Delta P_{0}$. Here $\alpha$ and $\beta$ are dimensionless, positive coefficients, and independent of $\Delta x$ and $\Delta P$ but may in general depend on the expectation values of $x$ and $p$. It is easy to show that

$$
\begin{aligned}
& \Delta x_{i} \geq \Delta x_{\min }=\frac{\hbar \alpha L_{P}\left(1-2 \sqrt{1-12 \alpha^{2} \beta^{2} L_{P}^{4}}\right)}{16 \alpha^{2} \beta^{2} L_{P}^{4}-1}, \\
& \Delta p_{i} \geq \Delta p_{\min }=\frac{\hbar \beta L_{P}\left(1+2 \sqrt{1-12 \alpha^{2} \beta^{2} L_{P}^{4}}\right)}{16 \alpha^{2} \beta^{2} L_{P}^{4}-1} .
\end{aligned}
$$

In particular, we suppose that operators of position and momentum obey the following commutation relation:

$$
[x, p]=i \hbar\left(1-2 \alpha p+4 \alpha^{2} p^{2}+4 \beta^{2} x^{2}\right) \text {. }
$$

Then, we obtain

$$
\frac{\left(\alpha L_{P}+\Delta x_{i}\right)-\sqrt{\left(\alpha L_{P}+\Delta x_{i}\right)^{2}-\left(4 \alpha^{2} L_{P}{ }^{2}\right)\left(1+4 \beta^{2} L_{P}{ }^{2}\left(\Delta x_{i}\right)^{2}\right)}}{4 \alpha^{2} L_{P}{ }^{2}}
$$

$$
\leq \Delta p_{i}
$$

$$
\leq \frac{\left(\alpha L_{P}+\Delta x_{i}\right)+\sqrt{\left(\alpha L_{P}+\Delta x_{i}\right)^{2}-\left(4 \alpha^{2} L_{P}^{2}\right)\left(1+4 \beta^{2} L_{P}^{2}\left(\Delta x_{i}\right)^{2}\right)}}{4 \alpha^{2} L_{P}^{2}} .
$$

So, the curve on the boundary of the allowed region is given by

$$
\begin{aligned}
\Delta p_{i}= & \left(\frac{\alpha L_{P}+\Delta x_{i}}{4 \alpha^{2} L_{P}{ }^{2}}\right) \\
& \times\left(1-\sqrt{1-\frac{\left(4 \alpha^{2} L_{P}^{2}\right)\left(1+4 \beta^{2} L_{P}^{2}\left(\Delta x_{i}\right)^{2}\right)}{\left(\alpha L_{P}+\Delta x_{i}\right)^{2}}}\right) .
\end{aligned}
$$

\section{TeV-Scale Black Hole Thermodynamics with Minimal Length and Maximal Momentum (GUP*)}

3.1. Temperature of TeV-Scale Black Hole. The Hawking temperature for a spherically symmetric black hole has been obtained in several ways by using the GUP and general properties of black holes are studied in this framework (see, for instance, [10]). Based on the relation (1), a simple calculation, with stress on the correct limiting results, gives

$$
\Delta p_{i}=\left(\frac{\alpha L_{P}+2 \Delta x_{i}}{4 \alpha^{2} L_{P}^{2}}\right)\left[1-\sqrt{1-\frac{8 \alpha^{2} L_{P}^{2}}{\left(\alpha L_{P}+2 \Delta x_{i}\right)^{2}}}\right] .
$$

Following the heuristic argument of [10], based on the uncertainty principle, we have

$$
T_{H}=\frac{(d-3)}{2 \pi} \Delta p_{i}
$$

Through this paper the natural units are used so that $\hbar=$ $G_{4}=C=k_{B}=1$. The constant of $(14),(d-3) / 2 \pi$, is a calibration factor in $d$ dimensional space-time (see, for instance, $[59,60])$. So, the modified black hole temperature based on the GUP* becomes

$$
T=\frac{(d-3)}{2 \pi}\left(\frac{\alpha L_{P}+2 \Delta x_{i}}{4 \alpha^{2} L_{P}{ }^{2}}\right)\left(1-\sqrt{1-\frac{8 \alpha^{2} L_{P}{ }^{2}}{\left(\alpha L_{P}+2 \Delta x_{i}\right)^{2}}}\right) .
$$

In the vicinity of the black hole surface there is an inherent uncertainty in the position of any particle of about the Schwarzschild radius (see [61]):

$$
\begin{array}{r}
\Delta x_{i} \approx r_{s}=\left(\frac{16 \pi G_{d} M}{(d-2) \Omega_{d-2}}\right)^{1 /(d-3)}=\omega_{d} L_{P} m^{1 /(d-3)}, \\
m=\frac{M}{M_{p}} .
\end{array}
$$

where $\omega_{d}$ is the dimensionless area coefficient and is given by

$$
\omega_{d}=\left(\frac{16 \pi}{(d-2) \Omega_{d-2}}\right)^{1 /(d-3)}, \quad \Omega_{d-2}=\frac{2 \pi^{(d-1) / 2}}{\Gamma((d-1) / 2)} .
$$




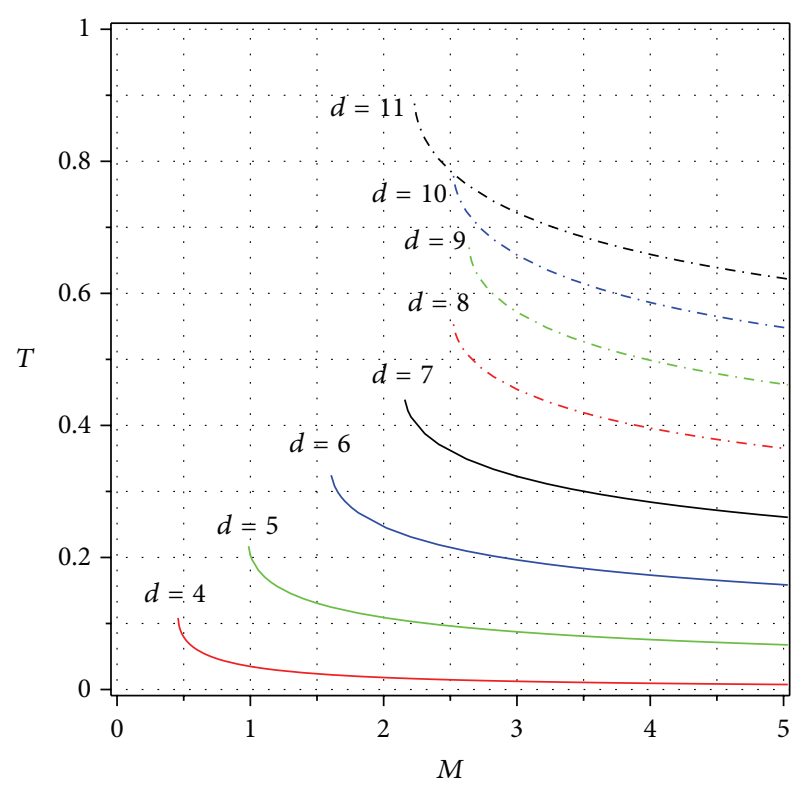

FIgURE 1: Temperature of a black hole as a function of mass, for different numbers of space-time dimensions with $\alpha=1$, and in the presence of GUP*. Mass is in the unit of the Planck mass and temperature is in the unit of the Planck energy.

We substitute (16) into (15) and obtain

$$
\begin{aligned}
T= & \left(\frac{d-3}{8 \pi L_{P}}\right)\left(\frac{\alpha+2 \omega_{d} m^{1 /(d-3)}}{\alpha^{2}}\right) \\
& \times\left(1-\sqrt{1-\frac{8 \alpha^{2}}{\left(\alpha+2 \omega_{d} m^{1 /(d-3)}\right)^{2}}}\right) .
\end{aligned}
$$

Based on this relation, GUP* gives rise to the existence of a minimal mass of a black hole given by

$$
M_{\min }=\left(\frac{\alpha}{\omega_{d}}\left(\sqrt{2}-\frac{1}{2}\right)\right)^{d-3} M_{p} .
$$

From this expression we observe that the $\mathrm{BH}$ temperature is only defined for $m \geq m_{\min }$. For a $\mathrm{BH}$ with a mass equal to $m_{\text {min }}$, the Hawking temperature reaches a maximum value given by

$$
T_{\max }=\frac{(d-3)}{\sqrt{8} \pi \alpha L_{P}} M_{p}^{1 /(d-3)} .
$$

This feature leads us to a finite temperature at the final stage of the black hole evaporation via Hawking radiation.

By Figure 1, the evaporation process of $\mathrm{TeV}$-scale Schwarzschild black hole in the framework of GUP* is commonly organized in two stages: in the first stage black hole temperature increases through its evaporation process. This phase is known as the Hawking phase, lasting up to the time in which it comes near to a maximum value which is known as the halt phase. In this stage, the temperature reaches

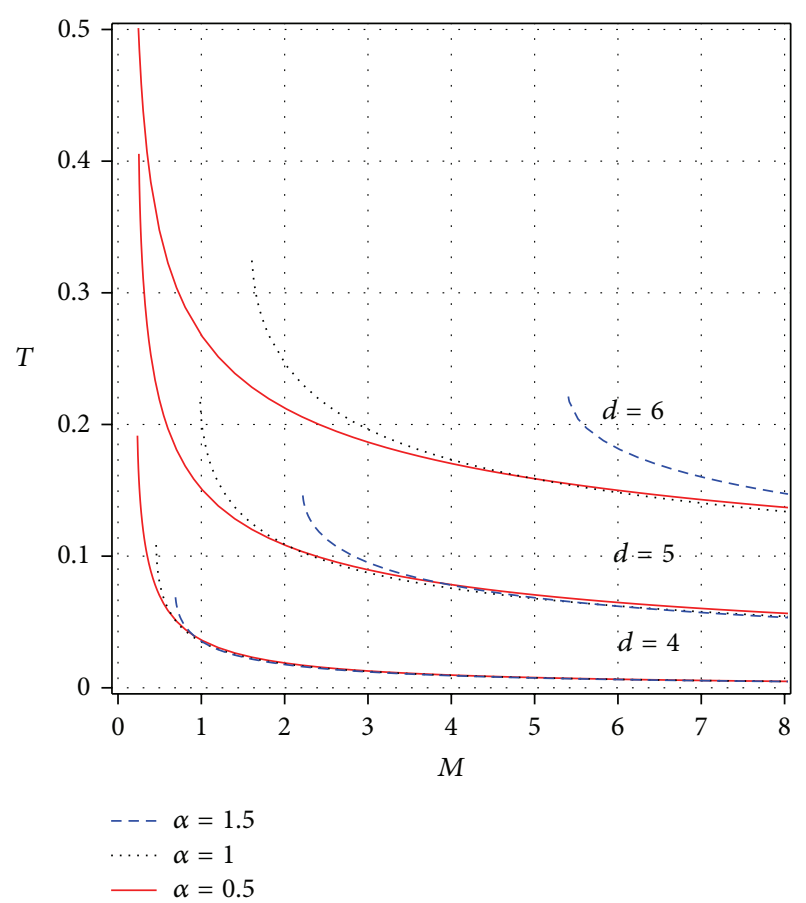

FIGURE 2: Black hole temperature as a function of mass for arbitrary numbers of space-time dimensions and in presence of GUP*. On the left hand side of the figure, from bottom to top, the curves are corresponding to $\alpha=0.5,1$, and 1.5 , respectively.

to a nonzero, UV cutoff case with a finite nonzero mass which is known as a Planck-size remnant (see, for instance, $[10,62-$ 65]). Also, as Figure 2 shows, when $\alpha$ increases, the order-ofmagnitude of the minimum mass increases and the location of the temperature peak displaces to the lower temperatures.

3.2. Entropy and Heat Capacity of TeV-Scale Black Hole. To obtain an analytic form of the black hole entropy with GUP*, let us acquire a Taylor expansion of (22) around $\alpha=0$ :

$$
\begin{aligned}
T= & \left(\frac{d-3}{8 \pi L_{P}}\right)\left(\frac{\alpha+2 \omega_{d} m^{1 /(d-3)}}{\alpha^{2}}\right) \\
& \times\left[1-\left(1-\frac{\alpha^{2}}{\omega_{d}^{2} m^{2 /(d-3)}}+\frac{\alpha^{3}}{\omega_{d}^{3} m^{3 /(d-3)}}+O\left(\alpha^{4}\right)\right)\right] .
\end{aligned}
$$

This relation can be rewritten as follows:

$$
T=\left(\frac{d-3}{8 \pi L_{P}}\right)\left[\frac{2}{\omega_{d} m^{1 /(d-3)}}-\frac{\alpha}{\omega_{d}^{2} m^{2 /(d-3)}}\right] .
$$

The entropy is obtained by the integration of $d S=c^{2} d M / T$ and we obtain a modified GUP* entropy from (22) accordingly:

$$
S=\int_{M_{\min }}^{M} d M\left(\frac{8 \pi L_{P}}{d-3}\right)\left[\frac{2}{\omega_{d} m^{1 /(d-3)}}-\frac{\alpha}{\omega_{d}^{2} m^{2 /(d-3)}}\right]^{-1} .
$$


By this manner, we have accepted that black hole entropy reaches to zero at $M_{\text {min }}$, where the $\mathrm{BH}$ mass is minimized with a radius of the order of the Planck length. Hawking evaporation has to stop once the black hole reduces to a Planck size remnant. In fact, it is impossible for a microblack hole to have the mass less than the $M_{\text {min }}$. These Planck size remnants can be considered as a possible candidate for dark matter. Finally, we calculate the integral for some values of $d$. We obtain for $d=4,5,6$ the following expressions, respectively, supposing $M_{P}=1 \mathrm{TeV}$.

For $d=4$,

$$
\begin{aligned}
S_{4}= & 2 \pi L_{P}\left[\left(\omega_{d} m^{2}+\alpha m\right)-\frac{a^{2}(8-\sqrt{8})}{4 \omega_{d}}\right] \\
& +\frac{\pi L_{P} a^{2}}{\omega_{d}} \ln \left(\frac{\alpha-2 \omega_{d} m}{(2-\sqrt{2}) \alpha}\right) .
\end{aligned}
$$

For $d=5$,

$$
\begin{aligned}
S_{5}= & \frac{4}{3} \pi \omega_{d} L_{P}\left[m^{3 / 2}-\left(\frac{\alpha}{2 \omega_{d}}(\sqrt{2}-1)\right)^{3}\right] \\
& +\pi L_{P} \alpha\left[m-\left(\frac{\alpha}{2 \omega_{d}}(\sqrt{2}-1)\right)^{2}\right] \\
& +\frac{\pi L_{P} \alpha^{2}}{\omega_{d}}\left[\sqrt{m}-\frac{\alpha}{2 \omega_{d}}(\sqrt{2}-1)\right] \\
& +\frac{\pi L_{P} a^{3}}{2 \omega_{d}^{2}} \ln \left(\frac{\alpha-2 \omega_{d} \sqrt{m}}{(2-\sqrt{2}) \alpha}\right) .
\end{aligned}
$$

For $d=6$,

$$
\begin{aligned}
S_{6}= & \pi L_{P} \omega_{d}\left(m^{4 / 3}-\left(\frac{\alpha}{2 \omega_{d}}(\sqrt{2}-1)\right)^{4}\right) \\
& +\frac{2}{3} \pi L_{P} a\left(m-\left(\frac{\alpha}{2 \omega_{d}}(\sqrt{2}-1)\right)^{3}\right) \\
& +\frac{\pi L_{P} a^{2}}{2 \omega_{d}}\left(m^{2 / 3}-\left(\frac{\alpha}{2 \omega_{d}}(\sqrt{2}-1)\right)^{2}\right) \\
& +\frac{\pi L_{P} \alpha^{3}}{2 \omega_{d}^{2}}\left(\sqrt[3]{m}-\left(\frac{\alpha}{2 \omega_{d}}(\sqrt{2}-1)\right)\right) \\
& +\frac{\pi L_{P} \alpha^{4}}{4 \omega_{d}^{3}} \ln \left(\frac{\alpha-2 \omega_{d} m^{1 / 3}}{(2-\sqrt{2}) \alpha}\right) .
\end{aligned}
$$

Now we see that the coefficient of the logarithm is obtained as a positive number for all extra dimensions (see also [11, 66-72]). We estimate the logarithmic correction term in extra dimensional model based on GUP* and it can be given by

$$
S_{d}^{(\log )} \approx \frac{\pi L_{P} \alpha^{d-2}}{2^{d-4} \omega_{d}^{d-3}} \ln \left(\frac{\alpha-2 \omega_{d} m^{1 /(d-3)}}{(2-\sqrt{2}) \alpha}\right) .
$$

We can also calculate the relations between event horizon area and entropy of a large $\mathrm{BH}$ easily (see, for instance, [11]). The result is shown in Figure 3. This figure shows that in scenarios with extra dimensions, black hole entropy decreases. The classical picture breaks down since the degrees of freedom of the black hole, that is, its entropy, are small. In this situation one can use the semiclassical entropy to measure the validity of the semiclassical approximation. Therefore, higher dimensional black hole remnants have less classical features relative to their four dimensional counterparts. To calculate the heat capacity of a black hole, we use a well-known thermodynamical relation $C=T(d S / d T)=$ $d M / d T$. So we obtain the heat capacity as a function of mass $M$ as follows:

$$
\begin{aligned}
C= & \left(\frac{4 \alpha^{2} \pi L_{P} m^{(d-4) /(d-3)}}{\omega_{d}}\right) \\
& \times \frac{\sqrt{1-8 \alpha^{2} /\left(2 \omega_{d} L_{P} m^{1 /(d-3)}+\alpha\right)^{2}}}{\sqrt{1-8 \alpha^{2} /\left(2 \omega_{d} L_{P} m^{1 /(d-3)}+\alpha\right)^{2}}-1} .
\end{aligned}
$$

The result is shown in Figure 4. At the Planck mass the slope is infinite, corresponding to the zero heat capacity of the black hole.

\section{TeV-Scale Black Hole Thermodynamics with Minimal Length, Minimal Momentum, and Maximal Momentum (GUP ${ }^{* *}$ )}

4.1. Temperature of TeV-Scale Black Hole. In what follows, we use GUP** and calculate the temperature of TeV-scale black hole. Based on the relations (14) and (16), we obtain

$$
\begin{aligned}
T= & \frac{(d-3)}{2 \pi}\left(\frac{\alpha L_{P}+\Delta x_{i}}{4 \alpha^{2} L_{P}^{2}}\right) \\
& \times\left(1-\sqrt{1-\frac{\left(4 \alpha^{2} L_{P}^{2}\right)\left(1+4 \beta^{2} L_{P}^{2}\left(\Delta x_{i}\right)^{2}\right)}{\left(\alpha L_{P}+\Delta x_{i}\right)^{2}}}\right)
\end{aligned}
$$

or

$$
\begin{aligned}
T= & \left(\frac{d-3}{8 \pi L_{P}}\right)\left(\frac{\alpha+\omega_{d} m^{1 /(d-3)}}{\alpha^{2}}\right) \\
& \times\left(1-\sqrt{1-\frac{4 \alpha^{2}\left(1+4 \beta^{2} L_{P}^{2}\left(\omega_{d} L_{P} m^{1 /(d-3)}\right)^{2}\right)}{\left(\alpha+\omega_{d} m^{1 /(d-3)}\right)^{2}}}\right) .
\end{aligned}
$$

Therefore, the uncertainty relation $\mathrm{GUP}^{* *}$ gives rise to the existence of a minimal mass of a black hole as

$$
M_{\min }=\left(\frac{\alpha}{\omega_{d}}\left(\frac{1 \mp 2 \sqrt{1-12 \alpha^{2} \beta^{2} L_{P}^{4}}}{16 \alpha^{2} \beta^{2} L_{P}^{4}-1}\right)\right)^{d-3} M_{p} .
$$




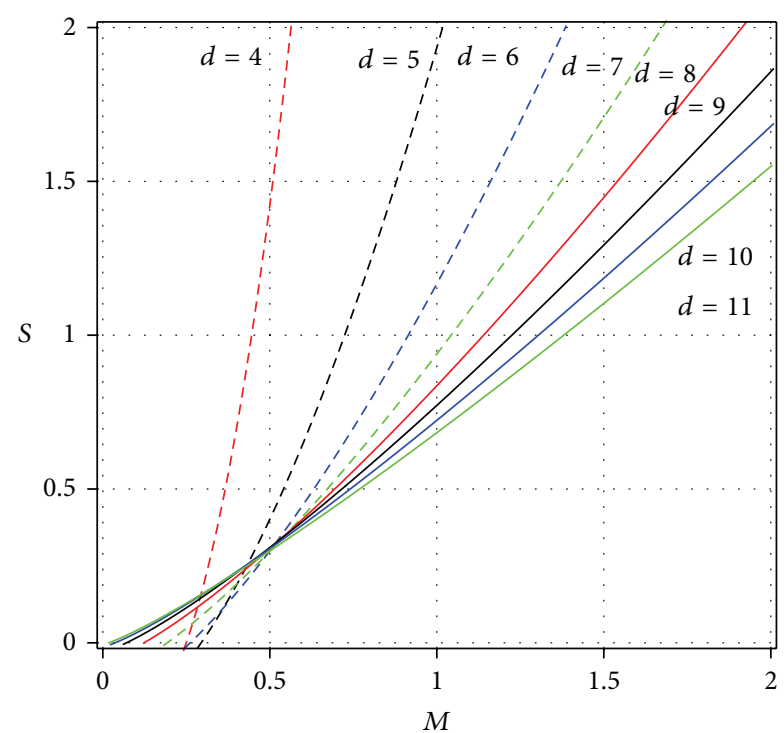

FIGURE 3: Black hole entropy as a function of mass for different numbers of space-time dimensions in the presence of GUP*

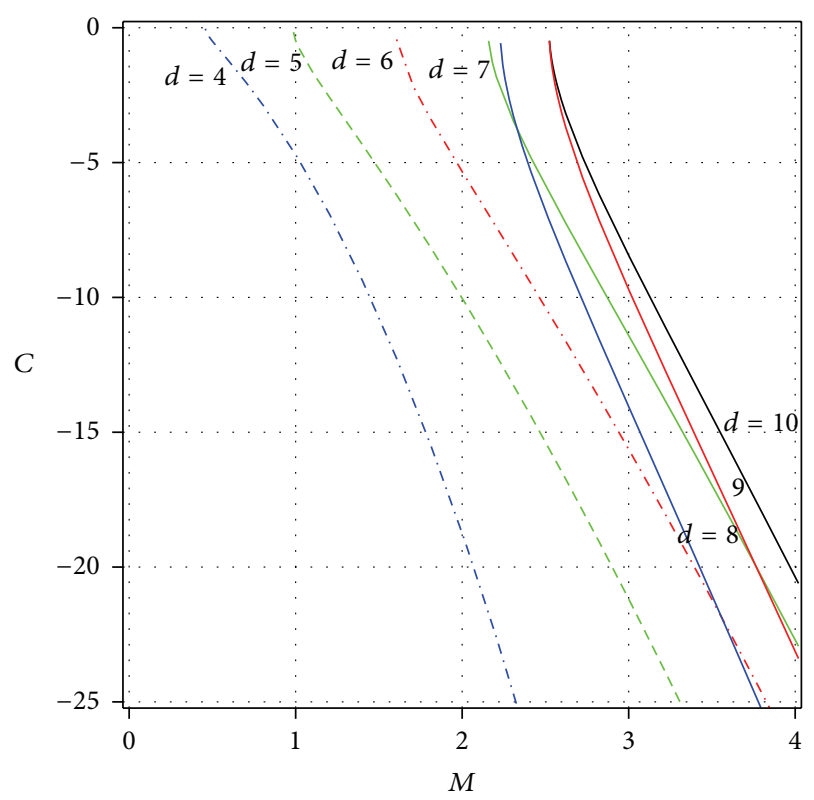

FIGURE 4: Black hole heat capacity as a function of mass for different numbers of space-time dimensions with $\alpha=1$ in the presence of GUP* .

This result agrees with the standard result for mass if the negative sign is chosen, whereas the positive sign has no evident physical meaning. Also, we restrict the range of the parameter $\alpha$ to be $\alpha^{2} \beta^{2} \leq 1 / 12 L_{P}{ }^{4}$ and $\alpha^{2} \beta^{2} \neq 1 / 16 L_{P}{ }^{4}$, for $\alpha \geq 0, \beta \geq 0$. This shows that $\alpha$ and $\beta$ cannot take any arbitrary value and this point should be considered in the previous equation. We can also conclude that $\alpha$ and $\beta$ are related to each other and are not independent parameters.
Now, the finite temperature at the final stage of the Hawking radiation takes the following form:

$$
\begin{aligned}
T_{\max }= & \frac{(d-3)}{8 \pi \alpha L_{P}} \\
& \times\left(\frac{16 \alpha^{2} \beta^{2} L_{P}{ }^{4}-2 \sqrt{1-12 \alpha^{2} \beta^{2} L_{P}}}{16 \alpha^{2} \beta^{2} L_{P}{ }^{4}-1}\right) M_{P}{ }^{1 /(d-3)} .
\end{aligned}
$$

One can see that uncertainty relation GUP ${ }^{* *}$ causes increasing of the black holes temperature in comparison with relations (18) and (20). Let us investigate temperature as a function of $M$ to see the effect of GUP ${ }^{* *}$ in another fashion. One can find that temperature has an extremum point when the mass of the black hole reaches the following value for $d=4$ :

$$
\begin{aligned}
& M_{\text {ext }} \\
& =\alpha\left(1-\frac{1-12 \alpha^{2} \beta^{2} L_{p}{ }^{4}+32 \sqrt{\alpha^{6} \beta^{6} L_{p}{ }^{12}-12 \alpha^{8} \beta^{8} L_{p}{ }^{16}}}{4 \alpha^{2} \beta^{2} L_{p}{ }^{4}+1+64 \alpha^{4} \beta^{4} L_{p}{ }^{8}}\right) \\
& \times\left(\omega _ { d } \left(\frac{1-12 \alpha^{2} \beta^{2} L_{p}{ }^{4}+32 \sqrt{\alpha^{6} \beta^{6} L_{p}^{12}-12 \alpha^{8} \beta^{8} L_{p}^{16}}}{4 \alpha^{2} \beta^{2} L_{p}{ }^{4}+1+64 \alpha^{4} \beta^{4} L_{p}^{8}}\right.\right. \\
& \left.\left.+16 \alpha^{2} \beta^{2} L_{p}^{4}-1\right)\right)^{-1} .
\end{aligned}
$$

It is easy to see that at a point temperature of the black hole takes a minimal value when $M_{\text {ext }}$ is on the boundary of the allowed region and it is larger than $M_{\text {min }}$. We know that black hole thermodynamics is only defined for $M \geq M_{\min }$. Then, $M_{\text {ext }}$ is acceptable and has an evident physical meaning. When the black holes mass is above $M_{\text {ext }}$, temperature (30) increases with the mass rise. When the mass is below the $M_{\text {ext }}$ its reduction leads to increasing of the temperature. Similar mass-temperature dependence was obtained in case of Schwarzschild-AdS black hole with modified generalized principle (7). We note that minimal temperature is the consequence of a minimal uncertainty in momentum and it can appear for different black holes metrics. In Figure 5, we have compared the temperature of black hole as a function of the mass for $d=4$ and for different types of GUPs.

Figure 6 that follows from the above results can be interpreted as follows: a small black hole, with temperature greater than the ambient temperature, should radiate photons, as well as other ordinary particles, until it reaches Planck mass and size. It cannot radiate further and becomes an inert remnant, possessing only gravitational interactions.

4.2. Entropy and Heat Capacity of TeV-Scale Black Hole. In this section we proceed as in Section 2, but now with GUP ${ }^{* *}$, 


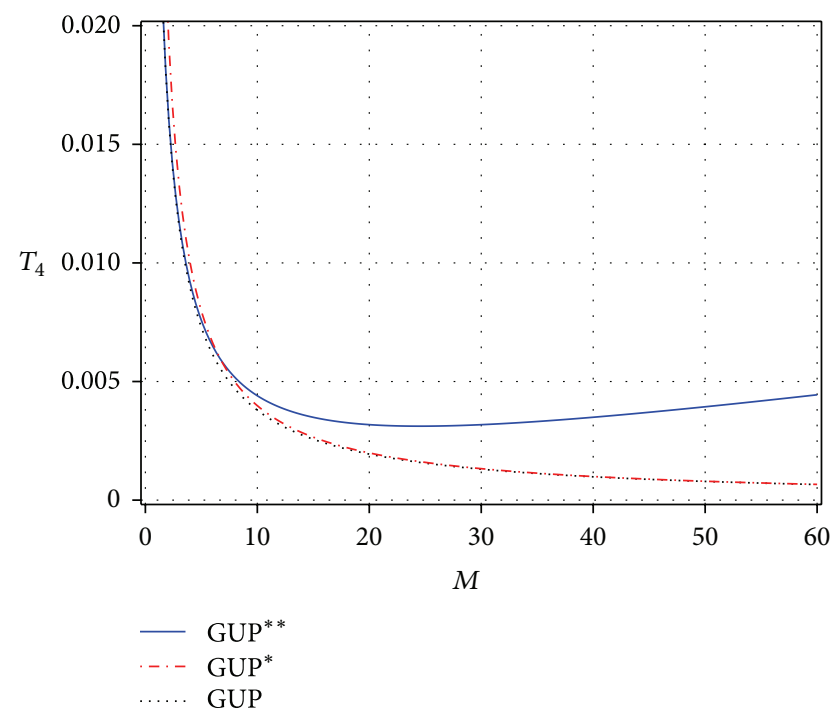

FIgURE 5: Temperature of black hole as a function of mass for $d=4$ and with three different types of GUPs.

and use the Taylor expansion to obtain an analytic form of the black hole entropy:

$$
\begin{aligned}
T=\left(\frac{d-3}{8 \pi L_{P}}\right) & \left(\frac{\alpha+\omega_{d} m^{1 /(d-3)}}{\alpha^{2}}\right) \\
& \times\left[1-\left(1-2 \frac{\left(m^{-2 /(d-3)}+4 \beta^{2} L_{P}^{4} \omega_{d}^{2}\right) \alpha^{2}}{\omega_{d}^{2}}\right.\right. \\
& +4 \frac{\left(m^{-3 /(d-3)}+4 m^{-1 /(d-3)} \beta^{2} L_{P}^{4} \omega_{d}^{2}\right) \alpha^{3}}{\omega_{d}^{3}} \\
& \left.\left.+O\left(\alpha^{4}\right)\right)\right] .
\end{aligned}
$$

This relation can be rewritten as follows:

$$
\begin{aligned}
T \approx & \frac{(d-3)}{4 \pi L_{P}} \\
& \times\left[\frac{1}{\omega_{d} m^{1 /(d-3)}}+4 \beta^{2} L_{P}^{4} \omega_{d} m^{1 /(d-3)}\right. \\
& \left.\quad-\left(\frac{1}{\omega_{d}^{2} m^{2 /(d-3)}}+4 \beta^{2} L_{P}^{4}\right) \alpha\right] .
\end{aligned}
$$

By integrating $d S=c^{2} d M / T$, we obtain

$$
\begin{aligned}
S=\int_{m_{\min }}^{m} d m\left(\frac{4 \pi L_{P}}{d-3}\right) & \\
\times & {\left[\frac{1}{\omega_{d} m^{1 /(d-3)}}+4 \beta^{2} L_{P}^{4} \omega_{d} m^{1 /(d-3)}\right.} \\
& \left.\quad-\left(\frac{1}{\omega_{d}^{2} m^{2 /(d-3)}}+4 \beta^{2} L_{P}^{4}\right) \alpha\right]^{-1} .
\end{aligned}
$$

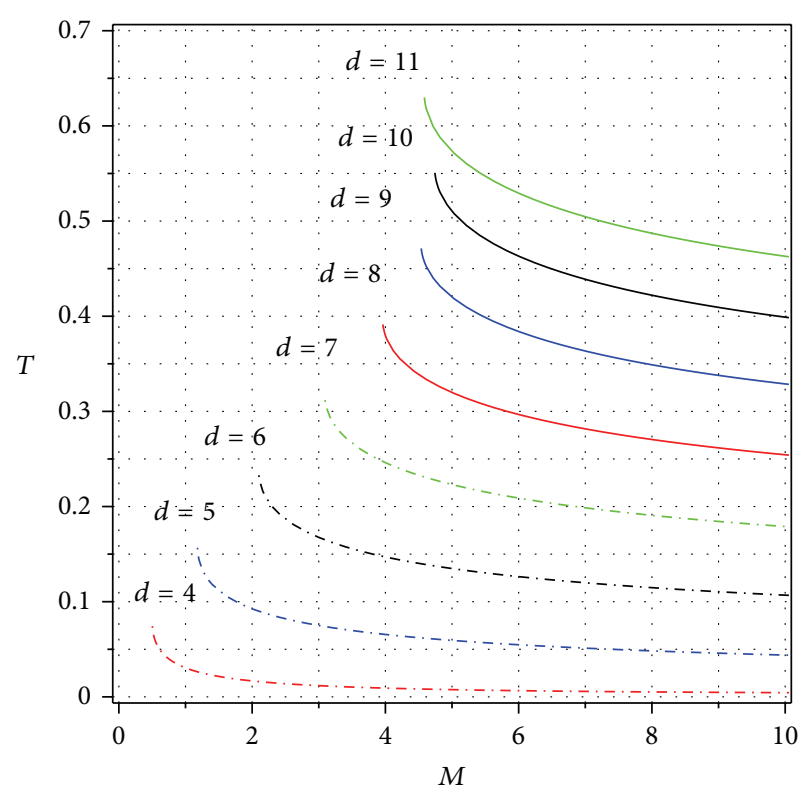

FIgURE 6: Temperature of a black hole as a function of mass with GUP $^{* *}$ and for different numbers of space-time dimensions with $\alpha=1$ and $\beta=0.01$. Mass is in the unit of the Planck mass and temperature is in the unit of the Planck energy.

The integral can be evaluated for given values of $d$ and we obtain the following for $d=4$, supposing $M_{p}=1 \mathrm{TeV}$ :

$$
\begin{gathered}
S_{4}=\left[\frac{4 \pi L_{P} \alpha^{2} \ln \left(m \omega_{d}-\alpha\right)}{\omega_{d}\left(1+4 \alpha^{2} \beta^{2} L_{P}^{4}\right)}\right. \\
+\frac{\pi \ln \left(1+4 m^{2} \beta^{2} L_{P}{ }^{4} \omega_{d}^{2}\right)}{2 L_{P}{ }^{3} \omega_{d}\left(1+4 \alpha^{2} \beta^{2} L_{P}{ }^{4}\right) \beta^{2}} \\
\left.+\frac{2 \pi \alpha \arctan \left(2 m \beta L_{P}{ }^{2} \omega_{d}\right)}{L_{P} \omega_{d}\left(1+4 \alpha^{2} \beta^{2} L_{P}{ }^{4}\right) \beta}\right]_{m_{\min }}^{m} .
\end{gathered}
$$

Using the value of $m_{\min }$, we obtain

$S_{4}$

$$
\begin{aligned}
= & \frac{4 \pi L_{P} \alpha^{2}}{\omega_{d}\left(1+4 \alpha^{2} \beta^{2} L_{P}{ }^{4}\right)} \\
& \times \ln \left(\frac{m \omega_{d}-\alpha}{\alpha\left(\left(1+2 \sqrt{1-12 \alpha^{2} \beta^{2} L_{P}{ }^{4}}\right) /\left(16 \alpha^{2} \beta^{2} L_{P}{ }^{4}-1\right)\right)-\alpha}\right) \\
& +\frac{\pi}{2 L_{P}{ }^{3} \omega_{d}\left(1+4 \alpha^{2} \beta^{2} L_{P}{ }^{4}\right) \beta^{2}} \\
& \times \ln \left(\frac{1+4 m^{2} \beta^{2} L_{P}{ }^{4} \omega_{d}{ }^{2}}{1+4\left(\left(1+2 \sqrt{1-12 \alpha^{2} \beta^{2} L_{P}}\right) /\left(16 \alpha^{2} \beta^{2} L_{P}{ }^{4}-1\right)\right)^{2} \alpha^{2} \beta^{2} L_{P}{ }^{4}}\right) \\
& +\frac{2 \pi \alpha}{L_{P} \omega_{d}\left(1+4 \alpha^{2} \beta^{2} L_{P}{ }^{4}\right) \beta} \\
& \times\left[\arctan \left(2 m \beta L_{P}{ }^{2} \omega_{d}\right)\right. \\
& \left.\quad-\arctan \left(2 \alpha \beta L_{P}{ }^{2}\left(\frac{1+2 \sqrt{1-12 \alpha^{2} \beta^{2} L_{P}}}{16 \alpha^{2} \beta^{2} L_{P}{ }^{4}-1}\right)\right)\right] .
\end{aligned}
$$


In a similar manner, for $d=5$, we find

$$
\begin{aligned}
S_{5}= & \frac{\pi \sqrt{m}}{L_{P}{ }^{3} \omega_{d} \beta^{2}}+4 \frac{\pi l \alpha^{3} \ln \left(\sqrt{m} \omega_{d}-\alpha\right)}{\omega_{d}^{2}\left(1+4 \alpha^{2} \beta^{2} L_{P}^{4}\right)} \\
& +\frac{1}{2} \frac{\pi \alpha \ln \left(1+4 m \beta^{2} L_{P}^{4} \omega_{d}^{2}\right)}{L_{P}{ }^{3} \omega_{d}^{2}\left(1+4 \alpha^{2} \beta^{2} L_{P}^{4}\right) \beta^{2}} \\
& -\frac{1}{2} \frac{\pi \arctan \left(2 \sqrt{m} \beta L_{P}^{2} \omega_{d}\right)}{L_{P}{ }^{5} \omega_{d}^{2}\left(1+4 \alpha^{2} \beta^{2} L_{P}^{4}\right) \beta^{3}} .
\end{aligned}
$$

Also for $d=6$, we have

$$
\begin{aligned}
S_{6}= & \frac{\pi m^{2 / 3}}{2 L_{P}{ }^{3} \omega_{d} \beta^{2}}+\frac{\pi \sqrt[3]{m} \alpha}{L_{P}{ }^{3} \omega_{d}^{2} \beta^{2}}+\frac{4 \pi L_{P} \alpha^{4} \ln \left(\sqrt[3]{m} \omega_{d}-\alpha\right)}{\omega_{d}^{3}\left(1+4 \alpha^{2} \beta^{2} L_{P}^{4}\right)} \\
& -\frac{\pi \ln \left(1+4 m^{2 / 3} \beta^{2} L_{P}^{4} \omega_{d}^{2}\right)}{8 L_{P}{ }^{7} \omega_{d}^{3}\left(1+4 \alpha^{2} \beta^{2} L_{P}^{4}\right) \beta^{4}} \\
& -\frac{\pi \alpha \arctan \left(2 \sqrt[3]{m} \beta L_{P}{ }^{2} \omega_{d}\right)}{2 L_{P}^{5} \omega_{d}^{3}\left(1+4 \alpha^{2} \beta^{2} L_{P}^{4}\right) \beta^{3}} .
\end{aligned}
$$

The important point here is the fact that the coefficient of the logarithm (logarithm prefactor) is obtained to be a positive number for all extra dimensions again. The logarithmic correction term in extra dimensional model based on GUP** is given by

$$
S_{d}{ }^{(\log )} \approx \frac{4 \pi L_{P} \alpha^{d-2}}{\omega_{d}^{d-3}\left(1+4 \alpha^{2} \beta^{2} L_{P}^{4}\right)} \ln \left(\omega_{d} m^{1 /(d-3)}-\alpha\right) .
$$

Figure 7 displays the black hole entropy versus its mass. The entropy reduces by increasing the number of the extra dimensions. In fact, as Figure 7 shows, black holes in extra dimensional models have numerically smaller entropy than black holes in four dimensions. Note that we have normalized the modified entropy to be zero at $M_{p}$.

Now we calculate the heat capacity of black hole as a function of its mass $M$. The result is

$$
\left.\left.\begin{array}{rl}
C= & \pi m \alpha^{2} L_{P}\left(\alpha+\omega_{d} m^{1 /(d-3)}\right) \\
& \times \sqrt{1-\frac{4 \alpha^{2}\left(1+4 \beta^{2} L_{P}{ }^{4} \omega_{d}{ }^{2} m^{2 /(d-3)}\right)}{\left(\alpha+\omega_{d} m^{1 /(d-3)}\right)^{2}}} \\
& \times\left(\sqrt{\omega_{d} m^{1 /(d-3)}\left(\alpha+\omega_{d} m^{1 /(d-3)}\right)}\right. \\
& \times\left(\sqrt{1-\frac{4 \alpha^{2}\left(1+4 \beta^{2} L_{P}{ }^{4} \omega_{d}{ }^{2} m^{2 /(d-3)}\right)}{\left(\alpha+\omega_{d} m^{1 /(d-3)}\right)^{2}}}-1\right)
\end{array}\right)\right]
$$

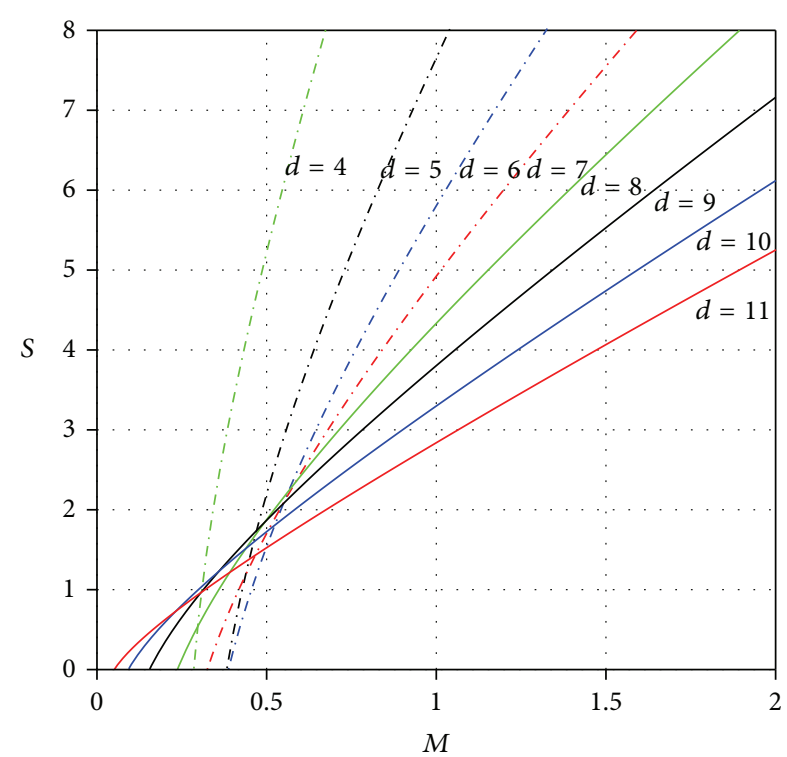

Figure 7: Black hole entropy as a function of mass for different numbers of space-time dimensions in presence of $\left(\mathrm{GUP}^{* *}\right)$.

When black hole mass is below the $M_{\text {ext }}$, the heat capacity is negative. It is equal to zero when the mass reaches $M_{\min }$. When the mass of the black hole is above the $M_{\text {ext }}$, the heat capacity is positive and tends to a finite value when mass goes to infinity. So $M_{\text {ext }}$ is the discontinuity point for the heat capacity. The behavior of black hole heat capacity as a function of mass for $d=4$ and with different types of GUPs is shown in Figure 8.

As can be seen in Figure 9, negative heat capacity shows that thermodynamical system is unstable and tends to decay. When heat capacity reaches zero, the system goes to stability. Indeed, black hole cannot radiate further and becomes an inert remnant, possessing only gravitational interactions.

Here we have shown that final stage of evaporation of a black hole is a remnant which has mass increasing with spacetime dimensions. One of the major problems with these remnants is the possibility of their detection. As interactions with black hole remnants are purely gravitational, the crosssection is extremely small, and direct observation of these remnants seems unlikely at least in the current situation. One possible indirect signature may be associated with the cosmic gravitational wave background.

\section{Conclusions and Discussion}

In this paper, using two different forms of the generalized uncertainty principles as our primary inputs, we have calculated the temperature and microcanonical entropy of a $\mathrm{TeV}$ scale black hole in the framework of the large extra dimensional scenarios. In scenarios with extra dimensions, black hole temperature increases with respect to the four dimensional ones (see Figures 1, 2, 5, and 6). This feature leads to faster decay and less classical behaviors for black holes. It is evident that in extra dimensional scenarios final stage of evaporation (black hole remnant) has mass more than its four 


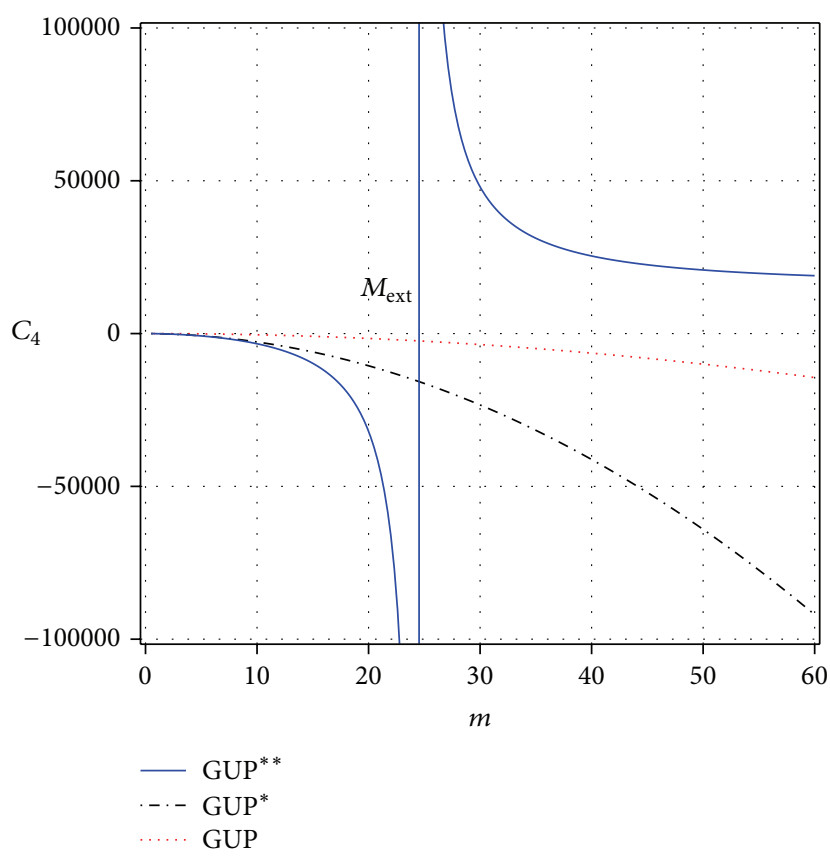

FIGURE 8: Black hole heat capacity as a function of mass for $d=4$ with different types of GUPs.

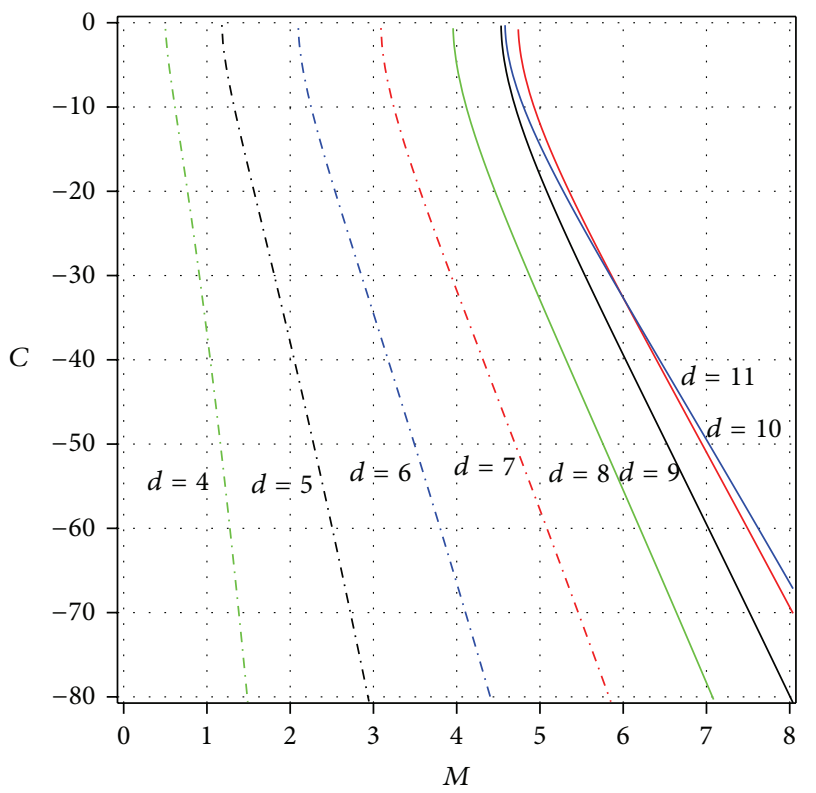

FIGURE 9: Black hole heat capacity as a function of mass with GUP** and for different numbers of space-time dimensions with $\alpha=1$ and $\beta=0.01$.

dimensional counterpart. In scenarios with extra dimensions, black hole entropy decreases (see Figures 3 and 7). The classical picture breaks down since the degrees of freedom of the black hole, that is, its entropy, are small. In this situation one can use the semiclassical entropy to measure the validity of the semiclassical approximation. It is evident that in extra dimensional scenarios final stage of evaporation (black hole
TABLE 1: GUP* ${ }^{*}$-corrected maximum temperature and minimum mass of $\mathrm{TeV}$ black hole for different values of parameter $\alpha$ in scenarios with large extra dimensions. Mass is in unit of the Planck mass and temperature is in unit of the Planck energy (supposing $\left.M_{p}=1 \mathrm{TeV}\right)$.

\begin{tabular}{lcccc}
\hline Case & $\alpha=0.5$ & $\alpha=1$ & $\alpha=0.5$ & $\alpha=1$ \\
& $M_{\min }$ & $M_{\min }$ & $T_{\max }$ & $T_{\max }$ \\
\hline$d=4$ & 0.22855 & 0.45710 & 0.22508 & 0.11254 \\
$d=5$ & 0.24615 & 0.98460 & 0.45015 & 0.22508 \\
$d=6$ & 0.20002 & 1.6002 & 0.67522 & 0.33761 \\
$d=7$ & 0.13465 & 2.1544 & 0.90030 & 0.4515 \\
$d=8$ & 0.078781 & 2.15210 & 1.1254 & 0.56269 \\
$d=9$ & 0.041247 & 2.6397 & 1.3504 & 0.67522 \\
$d=10$ & 0.019700 & 2.5216 & 1.5755 & 0.78766 \\
$d=11$ & 0.0087020 & 2.2278 & 1.5755 & 0.90030 \\
\hline
\end{tabular}

remnant) has event horizon area more than its four dimensional counterpart (see Figures 3 and 7). As an important issue, black hole radiation is mainly on the brane. In other words, black holes decay by emitting radiation mainly on the brane. This is in accordance with the results of [17].

The evaporation process for $\mathrm{TeV}$-scale black holes in the framework of the new-GUPs adopted in this paper is commonly organized in two stages: Hawking phase and halt phase. The corrections could be tested in current or future experiments at least by its prediction of black hole remnants. This remnant has been considered as a possible candidate to preserve information coming out of the black hole and therefore a possible solution of information loss problem [73]. The creation of the stable black hole remnants would prepare attractive new signatures which allows for the identification of such a remnant occurrence at colliders (such as the LHC) or Ultra High Energy Cosmic Ray (UHECR) [17] experiments. We obtained a numeric value for the new-GUP parameter. The existence and possible numerical value of this coefficient (logarithmic perfector) has been the basis of several interesting research programs in recent years [66-72].

One may urge that $\alpha$ and $\beta$ are constant quantities and their variations have no sense. We note, however, that $\alpha$ and $\beta$ are essentially model-dependent parameters; they depend on the aspects of the candidates for quantum gravity proposal. So, we conclude that $\alpha$ and $\beta$ are related nontrivially and can attain different values in different alternative approaches to quantum gravity.

As we have shown in Table $1, M_{\min }$ and $T_{\max }$ depend on the coefficient $\alpha$ and the space-time dimensions $d$ in the framework of the GUP* . Also, Table 1 in the framework of GUP* gives maximum temperature and minimum mass of the black hole remnant for different values of $d$ ranging from 4 to 11. If GUP* -parameter increases from left to right (for fixed $d$ ), the maximum temperature decreases and the minimum mass of the remnant increases. According to this table, as $d$ increases from top to bottom (for fixed $\alpha$ and $\beta$ ), the maximum temperature increases but minimum mass has a different behavior for different amounts of the coefficient. If $\alpha=0.5$, then the minimum mass enlarges up to $d=5$ and 
TABLE 2: GUP ${ }^{* *}$-corrected maximum temperature and minimum mass of black hole for different values of parameters $\alpha$ and $\beta$ in scenarios with large extra dimensions. Mass is in unit of the Planck mass and temperature is in unit of the Planck energy (supposing $M_{p}=1$ ).

\begin{tabular}{lcccc}
\hline Case & $\alpha=0.5, \beta=0.01$ & $\alpha=1, \beta=0.01$ & $\alpha=0.5, \beta=0.01$ & $T_{\max }$ \\
& $M_{\min }$ & $M_{\min }$ & 0.039789 & 0.039789 \\
$d=4$ & 0.2502 & 0.50020 & 0.079578 & 0.079578 \\
$d=5$ & 0.29458 & 1.1790 & 0.11937 & 0.11937 \\
$d=6$ & 0.26188 & 2.0969 & 0.15916 & 0.15916 \\
$d=7$ & 0.19284 & 3.0892 & 0.19894 & 0.19894 \\
$d=8$ & 0.12343 & 3.9558 & 0.23873 & 0.23873 \\
$d=9$ & 0.070694 & 4.5325 & 0.27852 & 0.27852 \\
$d=10$ & 0.036937 & 4.7380 & 0.31831 & 0.31831 \\
$d=11$ & 0.017850 & 4.5807 &
\end{tabular}

for $d \geq 6$, the minimum value of the remnant mass reduces. For $\alpha=1$, the minimum mass enlarges up to $d=10$ and for $d \geq 11$, the minimum value of the remnant mass reduces and so on. The situation is the same for GUP** as Table 2 shows. For example, for $\alpha=0.5$ and $\beta=0.01$ we see the same behavior as GUP* . But the numerical values of temperature and mass are different with the GUP* case.

We conclude that the minimal black hole mass becomes smaller by increasing the space-time dimension, $d$. This issue is very important because a black hole in model universes with extra dimensions at fixed $\alpha$ and $\beta$ can be produced at the LHC or future (next generation) accelerators just in the circumstance that $E_{\mathrm{cm}} \geq M_{\mathrm{min}}$, where $E_{\mathrm{cm}}$ is partonparton center-of-mass energy which is equal to $14 \mathrm{TeV}$. As we can see from (10), the minimal mass, $M_{\text {min }}$, relies efficiently upon the GUP parameter and the space-time dimension $d$. Therefore, in theories with large extra dimensions, if the number of space-time dimensions is adequately great, with an adequately small GUP parameter, the GUP* and GUP** slightly suppress the production of black holes in colliders such as the LHC. For an effectively large new-GUP parameter, the minimum value of mass enlarges by increasing $d$ without any bound. Therefore, in the new-GUP framework with sufficiently large $\alpha$ and $\beta$, the likelihood of the black hole production defeats at the LHC energies. So, black holes might not be observable in the LHC experiments while they could be still at the reach of the ultra-high energy cosmic ray events (see also $[64,65]$ ). Finally, we note that the generalized uncertainty principle and model universes with large extra dimensions are two well-established concepts in the modern theoretical physics. Phenomenologically, these concepts have important implications on the high energy physics problems such as production and evaporation processes of black holes. In scenarios with large extra dimensions, it is feasible for this scale to be not far from $\sim \mathrm{TeV}$ which directs to the famous hierarchy problem. If the minimal GUP mass as a remnant of the black hole is indeed of the order of $1 \mathrm{TeV}$, then it seems that TeV scale black holes may be formed at the LHC. However, the size of the remnant depends sensitively on the GUP parameter and number of extra dimensions.

\section{Conflict of Interests}

The authors declare that there is no conflict of interests regarding the publication of this paper.

\section{Acknowledgment}

The work of H. Soltani is financially supported by the research council of the Islamic Azad University, Nour Branch, Nour, Iran.

\section{References}

[1] G. Veneziano, "A stringy nature needs just two constants," Europhysics Letters, vol. 2, no. 3, p. 199, 1986.

[2] A. Kempf, G. Mangano, and R. B. Mann, "Hilbert space representation of the minimal length uncertainty relation," Physical Review D: Particles, Fields, Gravitation and Cosmology, vol. 52, no. 2, pp. 1108-1118, 1995.

[3] A. Kempf and G. Mangano, "Minimal length uncertainty relation and ultraviolet regularization," Physical Review D: Particles, Fields, Gravitation and Cosmology, vol. 55, no. 12, pp. 79097920, 1997.

[4] D. Amati, "Can spacetime be probed below the string size?" Physics Letters B, vol. 216, no. 1-2, pp. 41-47, 1989.

[5] L. J. Garay, "Quantum gravity and minimum length," International Journal of Modern Physics A, vol. 10, no. 2, p. 145, 1995.

[6] M. Maggiore, "Quantum groups, gravity, and the generalized uncertainty principle," Physical Review D, vol. 49, no. 10, pp. 5182-5187, 1994.

[7] K. A. Meissner, "Black-hole entropy in loop quantum gravity," Classical and Quantum Gravity, vol. 21, no. 22, pp. 5245-5251, 2004.

[8] F. Scardigli, "Generalized uncertainty principle in quantum gravity from micro-black hole gedanken experiment," Physics Letters B, vol. 452, pp. 39-44, 1999.

[9] R. J. Adler, "Six easy roads to the Planck scale," American Journal of Physics, vol. 78, no. 9, pp. 925-932, 2010.

[10] R. J. Adler, P. Chen, and D. I. Santiago, "The generalized uncertainty principle and black hole remnants," General Relativity and Gravitation, vol. 33, no. 12, pp. 2101-2108, 2001. 
[11] K. Nozari and A. S. Sefidgar, "On the existence of the logarithmic correction term in black hole entropy-area relation," General Relativity and Gravitation, vol. 39, no. 4, pp. 501-509, 2007.

[12] K. Nozari, "A possible mechanism for production of primordial black holes in early universe," Astroparticle Physics, vol. 27, no. 2-3, pp. 169-173, 2007.

[13] A. J. M. Medved, "A comment on black hole entropy or does nature abhor a logarithm?" Classical and Quantum Gravity, vol. 22, no. 1, pp. 133-142, 2005.

[14] A. J. M. Medved and E. C. Vagenas, "When conceptual worlds collide: the generalized uncertainty principle and the Bekenstein-Hawking entropy," Physical Review D, vol. 70, Article ID 124021, 2004.

[15] A. J. M. Medved, "Quantum-corrected entropy for (1+1)-dimensional gravity revisited," Classical and Quantum Gravity, vol. 20, no. 11, pp. 2147-2156, 2003.

[16] G. Gour and A. J. M. Medved, "Thermal fluctuations and blackhole entropy," Classical and Quantum Gravity, vol. 20, no. 15, pp. 3307-3326, 2003.

[17] J. L. Feng and A. D. Shapere, "Black hole production by cosmic rays," Physical Review Letters, vol. 88, Article ID 021303, 2002.

[18] H. Ohanian and R. Ruffini, Gravitation and Spacetime, 2nd edition, 1994.

[19] W. Kim, E. J. Son, and M. Yoon, "Thermodynamics of a black hole based on a generalized uncertainty principle," Journal of High Energy Physics, vol. 1, p. 035, 2008.

[20] M. Stetsko, "Microscopic black hole and uncertainty principle with minimal length and momentum," International Journal of Modern Physics A, vol. 28, no. 10, Article ID 1350029, 2013.

[21] K. Nouicer, "Black hole thermodynamics to all orders in the Planck length in extra dimensions," Classical and Quantum Gravity, vol. 24, no. 23, pp. 5917-5934, 2007.

[22] D. V. Fursaev, "Temperature and entropy of a quantum black hole and conformal anomaly," Physical Review D, vol. 51, no. 10, pp. R5352-R5355, 1995.

[23] R. B. Mann and S. N. Solodukhin, "Universality of quantum entropy for extreme black holes," Nuclear Physics B, vol. 523, no. 1-2, pp. 293-307, 1998.

[24] R. K. Kaul and P. Majumdar, "Logarithmic correction to the Bekenstein-Hawking entropy," Physical Review Letters, vol. 84, no. 23, pp. 5255-5257, 2000.

[25] N. Arkani-Hamed, S. Dimopoulos, and G. Dvali, “The hierarchy problem and new dimensions at a millimeter," Physics Letters B, vol. 429, no. 3-4, pp. 263-272, 1998.

[26] P. C. Argyres, S. Dimopoulos, and J. March-Russell, "Black holes and sub-millimeter dimensions," Physics Letters B, vol. 441, no. 1-4, pp. 96-104, 1998.

[27] R. Emparan, G. T. Horowitz, and R. C. Myers, "Black holes radiate mainly on the brane," Physical Review Letters, vol. 85, no. 3, pp. 499-502, 2000.

[28] J. L. Hewett, B. Lillie, and T. G. Rizzo, "Black holes in many dimensions at the CERN Large Hadron Collider: testing critical string theory," Physical Review Letters, vol. 95, no. 26, Article ID 261603, 2005.

[29] S. Dimopoulos and G. Landsberg, "Black holes at the Large Hadron Collider," Physical Review Letters, vol. 87, no. 16, Article ID 161602, 2001.

[30] B. Koch, M. Bleicher, and S. Hossenfelder, "Black hole remnants at the LHC," Journal of High Energy Physics, vol. 10, p. 053, 2005.
[31] H. Stoecker J, "Mini black holes in the first year of the LHC: discovery through di-jet suppression, multiple mono-jet emission and ionizing tracks in ALICE," Journal of Physics G: Nuclear and Particle Physics, vol. 32, no. 12, p. S429, 2006.

[32] G. T. Rizzo, "Black hole production at the LHC: effects of voloshin suppression," Journal of High Energy Physics, vol. 2, p. 011, 2002.

[33] M. Cavaglia, R. Godang, L. M. Cremaldi, and D. J. Summers, "Signatures of black holes at the LHC," Journal of High Energy Physics, vol. 6, p. 055, 2007.

[34] P. Meade and L. Randall, "Black holes and quantum gravity at the LHC," Journal of High Energy Physics, vol. 5, p. 003, 2008.

[35] P. Kanti, "Black holes at the large Hadron collider," in Physics of Black Holes, vol. 769 of Lecture Notes in Physics, pp. 387-423, Springer, Berlin, Germany, 2009.

[36] R. Casadio, S. Fabi, and B. Harms, "Possibility of catastrophic black hole growth in the warped brane-world scenario at the LHC," Physical Review D, vol. 80, Article ID 084036, 2009.

[37] G. Dvali, C. Gomez, and S. Mukhanov, "Probing quantum geometry at LHC," Journal of High Energy Physics, vol. 2011, no. 2, pp. 1-12, 2011.

[38] D. M. Gingrich, "Noncommutative geometry inspired black holes in higher dimensions at the LHC," Journal of High Energy Physics, vol. 1105, p. 022, 2010.

[39] A. F. Ali, S. Das, and E. C. Vagenas, "Proposal for testing quantum gravity in the lab," Physical Review D: Particles, Fields, Gravitation and Cosmology, vol. 84, no. 4, Article ID 044013, 2011.

[40] S. Das and E. C. Vagenas, "Universality of quantum gravity corrections," Physical Review Letters, vol. 101, Article ID 221301, 2008.

[41] K. Nozari and A. Etemadi, "Minimal length, maximal momentum, and Hilbert space representation of quantum mechanics," Physical Review D, vol. 85, Article ID 104029, 2012.

[42] P. Pedram, K. Nozari, and S. H. Taheri, "The effects of minimal length and maximal momentum on the transition rate of ultra cold neutrons in gravitational field," Journal of High Energy Physics, vol. 1103, p. 093, 2011.

[43] K. Nozari and S. Saghafi, "Natural cutoffs and quantum tunneling from black hole horizon," Journal of High Energy Physics, vol. 11, p. 005, 2012.

[44] S. Basilakos, S. Das, and E. C. Vagenas, "Quantum gravity corrections and entropy at the Planck time," Journal of Cosmology and Astroparticle Physics, vol. 1009, p. 027, 2010.

[45] K. Nozari and P. Shahini, "TeV scale black holes thermodynamics with extra dimensions and quantum gravity effects," http://arxiv-web3.library.cornell.edu/abs/1206.5624.

[46] G. Amelino-Camelia, "Relativity in spacetimes with shortdistance structure governed by an observer-independent (Planckian) length scale," International Journal of Modern Physics D: Gravitation, Astrophysics, Cosmology, vol. 11, no. 1, pp. 35-59, 2002.

[47] G. Amelino-Camelia, "Special treatment," Nature, vol. 418, no. 6893, pp. 34-35, 2002.

[48] G. Amelino-Camelia, "Doubly-special relativity: first results and key open problems," International Journal of Modern Physics D: Gravitation, Astrophysics, Cosmology, vol. 11, no. 10, pp. 1643-1669, 2002.

[49] J. Kowalski-Glikman, "Introduction to doubly special relativity," in Planck Scale Effects in Astrophysics and Cosmology, J. Kowalski-Glikman and G. Amelino-Camelia, Eds., vol. 669 of Lecture Notes in Physics, pp. 131-159, 2005. 
[50] K. Imiłkowska and J. Kowalski-Glikman, "Doubly special relativity as a limit of gravity," in Special Relativity, J. Ehlers and C. Lämmerzahl, Eds., vol. 702 of Lecture Notes in Physics, pp. 279298, 2006.

[51] J. Magueijo and L. Smolin, "Lorentz invariance with an invariant energy scale," Physical Review Letters, vol. 88, Article ID 190403, 2002.

[52] J. Magueijo and L. Smolin, "Generalized Lorentz invariance with an invariant energy scale," Physical Review D, vol. 67, Article ID 044017, 2003.

[53] J. Magueijo and L. Smolin, "String theories with deformed energy-momentum relations, and a possible nontachyonic bosonic string," Physical Review D, vol. 71, no. 2, Article ID 026010, 2005.

[54] J. L. Cortés and J. Gamboa, "Quantum uncertainty in doubly special relativity," Physical Review D, vol. 71, no. 6, Article ID 065015, 2005.

[55] H. Hinrichsen and A. Kempf, "Maximal localization in the presence of minimal uncertainties in positions and in momenta," Journal of Mathematical Physics, vol. 37, no. 5, pp. 2121-2137, 1996.

[56] M. Zarei and B. Mirza, "Minimal uncertainty in momentum: the effects of IR gravity on quantum mechanics," Physical Review D: Particles, Fields, Gravitation and Cosmology, vol. 79, no. 12, Article ID 125007, 2009.

[57] K. Nozari, F. Moafi, and F. Rezaee Balef, "Some aspects of supersymmetric field theories with minimal length and maximal momentum," Advances in High Energy Physics, vol. 2013, Article ID 252178, 6 pages, 2013.

[58] M. Roushan and K. Nozari, "Heisenberg algebra in the Bargmann-Fock space with natural cutoffs," Advances in High Energy Physics, vol. 2014, Article ID 353192, 6 pages, 2014.

[59] M. Cavaglià and S. Das, "How classical are TeV-scale black holes?" Classical and Quantum Gravity, vol. 21, no. 19, pp. 45114522, 2004.

[60] M. Cavagli, S. Das, and R. Maartens, "Will we observe black holes at the LHC?" Classical and Quantum Gravity, vol. 20, p. L205, 2003.

[61] R. J. Adler and T. K. Das, "Charged-black-hole electrostatics," Physical Review D: Particles, Fields, Gravitation and Cosmology, vol. 14, no. 10, pp. 2474-2477, 1976.

[62] K. Nozari and S. H. Mehdipour, "Black holes remnants in extra dimensions and dark matter," International Journal of Modern Physics A, vol. 21, no. 23-24, pp. 4979-4992, 2006.

[63] K. Nozari and S. H. Mehdipour, "Hawking radiation as quantum tunneling from a noncommutative Schwarzschild black hole," Classical and Quantum Gravity, vol. 25, no. 17, Article ID 175015, 2008.

[64] K. Nozari and S. H. Mehdipour, "Parikh-Wilczek tunneling from noncommutative higher dimensional black holes," Journal of High Energy Physics, vol. 0903, p. 061, 2009.

[65] K. Nozari and S. H. Mehdipour, "Quantum gravity and recovery of information in black hole evaporation," Europhysics Letters, vol. 84, no. 2, Article ID 20008, 2008.

[66] J. Mureika, P. Nicolini, and E. Spallucci, "Could any black holes be produced at the LHC?” Physical Review D, vol. 85, Article ID 106007, 2012.

[67] L. Bellagamba, R. Casadio, R. di Sipio, and V. Viventi, "Black hole remnants at the LHC," http://arxiv.org/abs/1201.3208.

[68] M. Domagala and J. Lewandowski, "Black-hole entropy from quantum geometry," Classical and Quantum Gravity, vol. 21, no. 22, pp. 5233-5243, 2004.
[69] A. Ghosh and P. Mitra, "Log correction to the black hole area law," Physical Review D, vol. 71, no. 2, Article ID 027502, 2005.

[70] A. Ashtekar, J. Engle, and C. van den Broeck, "Quantum horizons and black-hole entropy: inclusion of distortion and rotation," Classical and Quantum Gravity, vol. 22, no. 4, pp. L27L34, 2005.

[71] D. Rideout and S. Zohren, "Evidence for an entropy bound from fundamentally discrete gravity," Classical and Quantum Gravity, vol. 23, no. 22, pp. 6195-6213, 2006.

[72] A. J. M. Medved and E. C. Vagenas, "On Hawking radiation as tunneling with logarithmic corrections," Modern Physics Letters A, vol. 20, no. 23, pp. 1723-1728, 2005.

[73] D. N. Page, "Information in black hole radiation," Physical Review Letters, vol. 71, no. 23, pp. 3743-3746, 1993. 

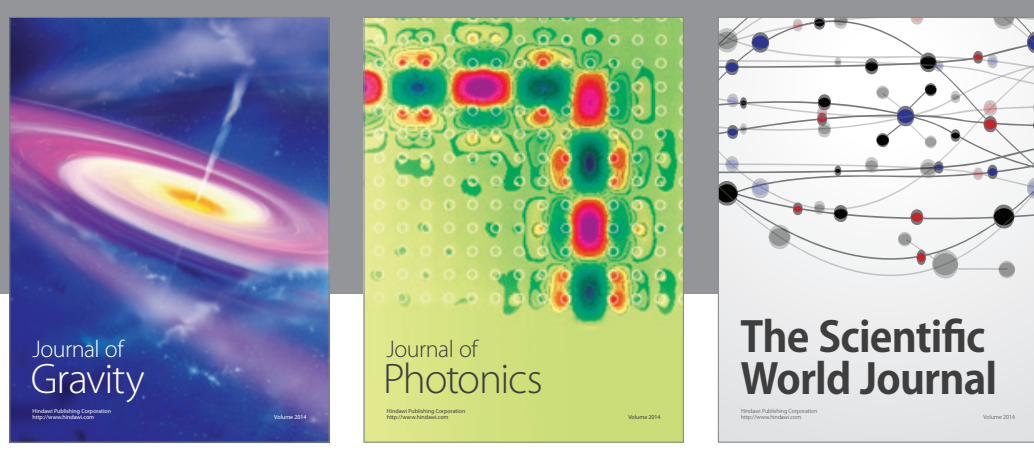

The Scientific World Journal
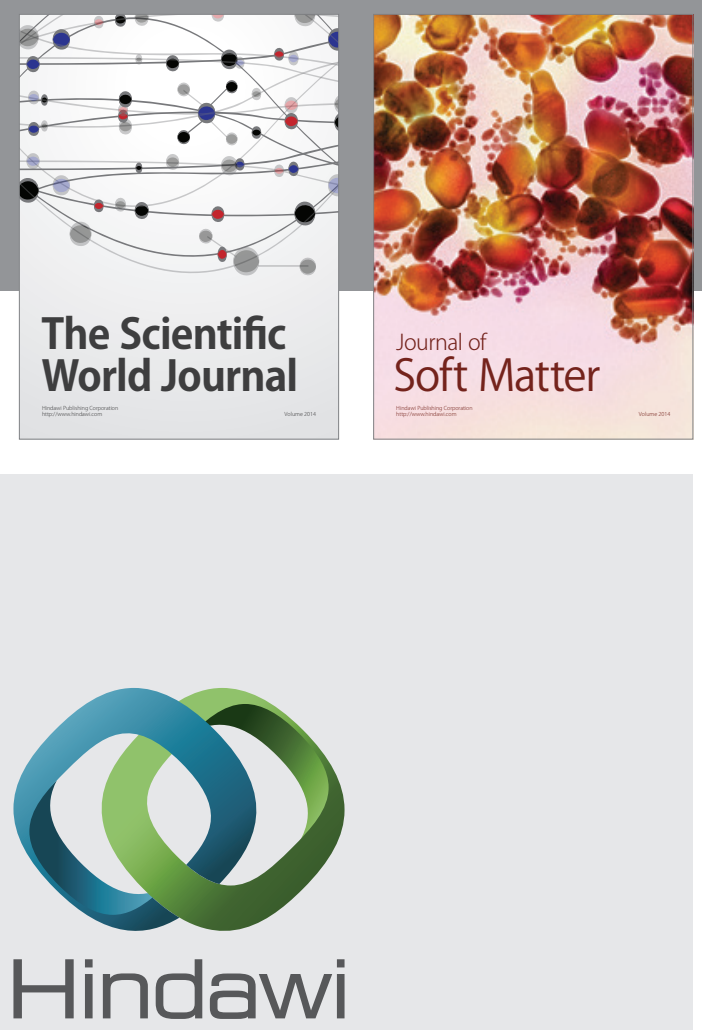

Submit your manuscripts at

http://www.hindawi.com

nternational Journal of

Statistical Mechanics
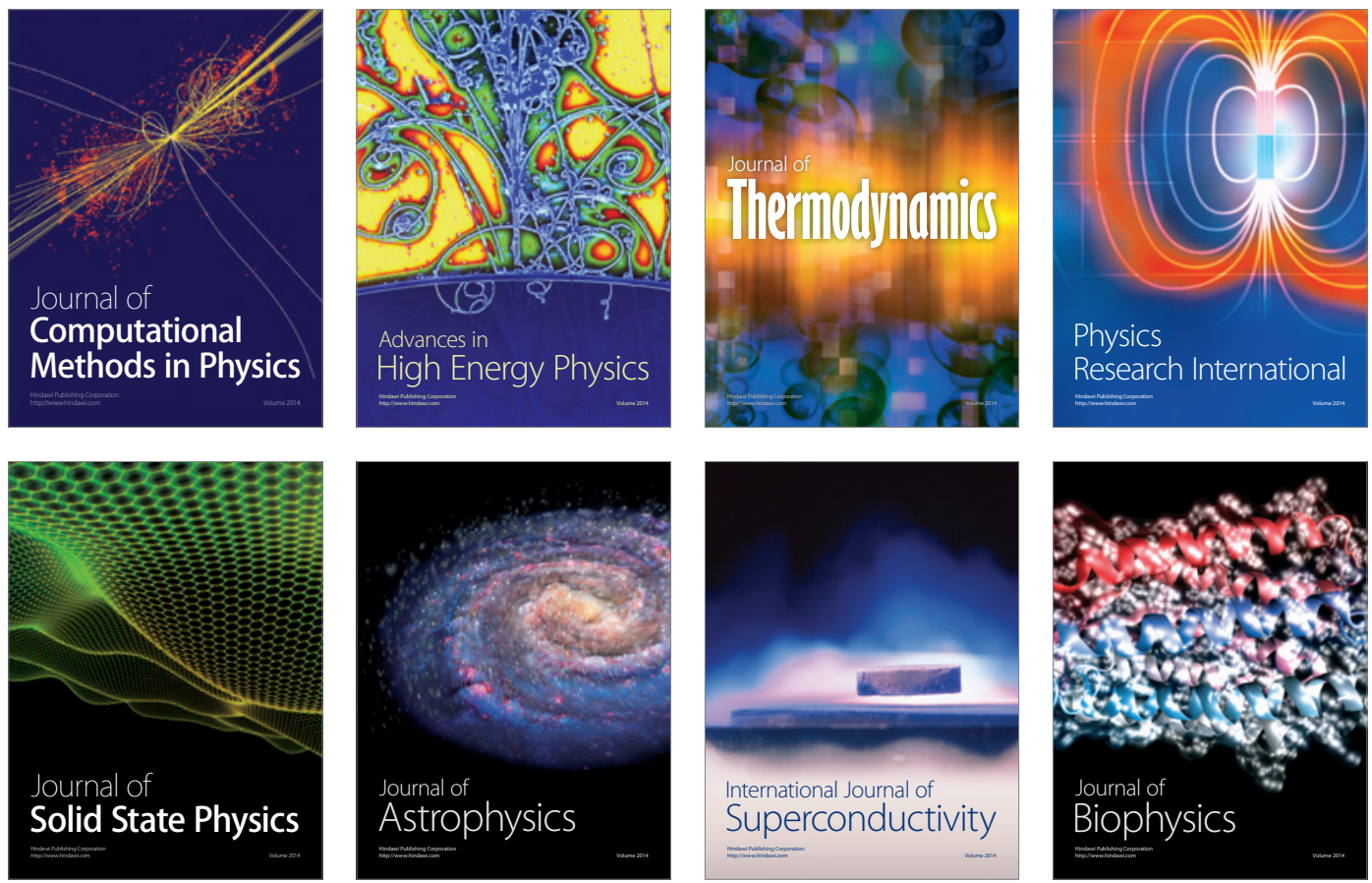
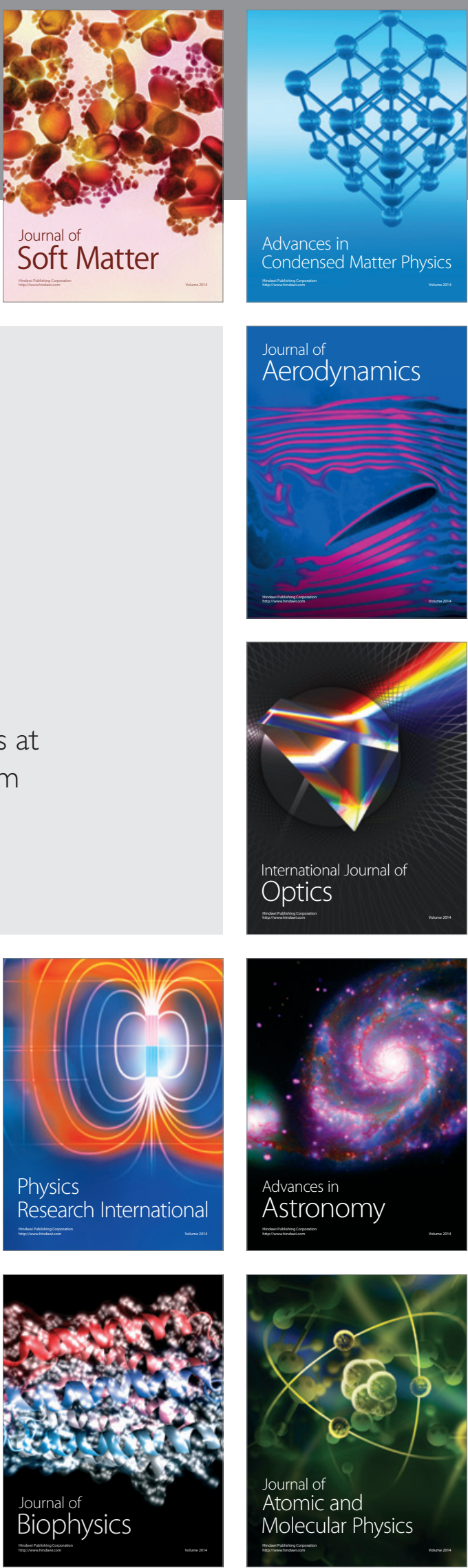